\title{
Identification in GRMD dog muscle of critical miRNAs involved in pathophysiology and effects associated with MuStem cell transplantation
}

Florence Robriquet ${ }^{1,2,3}$, Candice Babarit ${ }^{1,2}$, Thibaut Larcher ${ }^{1,2}$, Laurence Dubreil ${ }^{1,2}$, Mireille Ledevin ${ }^{1,2}$, Hélicia Goubin ${ }^{1,2}$, Karl Rouger ${ }^{1,2+}$ and Laëtitia Guével ${ }^{1,2,3^{*+}}$

\begin{abstract}
Background: Duchenne muscular dystrophy (DMD) is an X-linked muscle disease that leads to fibre necrosis and progressive paralysis. At present, DMD remains a lethal disease without any effective treatment, requiring a better understanding of the pathophysiological processes and comprehensive assessment of the newly identified therapeutic strategies. MicroRNAs including members of the muscle-specific myomiR family have been identified as being deregulated in muscle of DMD patients and in $m d x$ mice used as a model for DMD. In recent years, the Golden Retriever muscular dystrophy (GRMD) dog has appeared as the crucial animal model for objectively assessing the potential of new innovative approaches. Here, we first aim at establishing the muscle expression pattern of five selected miRNAs in this clinically relevant model to determine if they are similarly affected compared with other DMD contexts. Second, we attempt to show whether these miRNAs could be impacted by the systemic delivery of a promising stem cell candidate (referred to as MuStem cells) to implement our knowledge on its mode of action and/or identify markers associated with cell therapy efficacy.
\end{abstract}

Methods: A comparative study of miRNAs expression levels and cellular localization was performed on 9-monthold healthy dogs, as well as on three sub-sets of GRMD dog (without immunosuppression or cell transplantation, with continuous immunosuppressive regimen and with MuStem cell transplantation under immunosuppression), using RT-qPCR and in situ hybridization.

Results: We find that miR-222 expression is markedly up-regulated in GRMD dog muscle compared to healthy dog, while miR-486 tends to be down-expressed. Intriguingly, the expression of miR-1, miR-133a and miR-206 does not change. In situ hybridization exploration reveals, for the first time, that miR-486 and miR-206 are mainly localized in newly regenerated fibres in GRMD dog muscle. In addition, we show that cyclosporine-based immunosuppression, classically used in allogeneic cell transplantation, exclusively impacts the miR-206 expression. Finally, we demonstrate that intra-arterial administration of MuStem cells results in up-regulation of miR-133a and miR-222 concomitantly with a down-expression of two sarcomeric proteins corresponding to miR-222 targets.

\footnotetext{
* Correspondence: laetitia.guevel@univ-nantes.fr

Laëtitia Guével and Karl Rouger share equal contributions as senior authors.

${ }^{\dagger}$ Equal contributors

'INRA UMR 703 PAnTher "Physiopathologie Animale et bioThérapie du

muscle et du système nerveux", Oniris, Atlanpôle - La Chantrerie, Route du Gachet C.S. 40706, F-44307 Nantes Cedex 03, France

2LUNAM Université, Oniris, École nationale vétérinaire, agro-alimentaire et de

I'alimentation Nantes-Atlantique, F-44307 Nantes, France

Full list of author information is available at the end of the article
} 
(Continued from previous page)

Conclusion: We point out a differential muscle expression of miR-222 and miR-486 associated with the pathophysiology of the clinically relevant GRMD dog model with a tissue localization focused on regenerated fibres. We also establish a modified expression of miR-133a and miR-222 subsequent to MuStem cell infusion.

Keywords: miRNA, Stem cell therapy, Duchenne muscular dystrophy, GRMD dog, Skeletal muscle, In situ hybridization

\section{Background}

Duchenne muscular dystrophy (DMD) is a progressive and fatal X-linked recessive disorder of skeletal and cardiac muscles. It is a particularly severe and common form of muscular dystrophy, affecting one in 3500 males at birth [1]. Mutations in the gene encoding the dystrophin lead to a lack of this protein, which normally ensures the essential link between the subsarcolemmal cytoskeleton and the extracellular matrix at the muscle fibre membrane $[2,3]$. DMD is characterized by repeated cycles of necrosis/regeneration of muscle fibres, progressive replacement of skeletal muscle by fibrotic and adipose tissues and generalized muscle weakness, paralysis and death [4]. Recently, several gene and cellbased strategies have been developed to restore dystrophin expression in the Golden Retriever muscular dystrophy (GRMD) dog, the clinically relevant animal model of DMD. Some of these innovative approaches have now entered preclinical studies $[5,6]$. In parallel, numerous studies are ongoing to define muscle molecular signatures that could be used to characterize dystrophic dog tissue $[7,8]$ or to validate the effect of promising therapeutic strategies $[9,10]$.

MicroRNAs (miRNAs) are short non-coding RNA sequences of 21 to 25 nucleotides that regulate gene expression at a post-transcriptional level. Through binding to target mRNA, they promote its degradation or translational inhibition [11, 12]. In muscle, specific miRNAs (known as myomiRs), such as miR-1, miR-133 and miR206 , are involved in regulation of the proliferation or differentiation of myogenic cells [13-16] and are especially regulated by transcription factors implicated in muscle growth and development $[17,18]$. Other miRNAs, such as miR-29, miR-34, miR-222 and miR-486, also play keyroles in modulating important pathways of skeletal muscle processes [19-22]. Over the last few years, miRNAs have been found to be deregulated in muscular dystrophies [23, 24]. A specific DMD signature has been identified based on eleven miRNAs that are deregulated both in $m d x$ mice and DMD patients [22]. As regards myomiRs, several studies report that miR-1 and miR-133 are under-expressed, while miR-206 is over-expressed in $m d x$ muscles [25-27]. All these findings indicate an important role of miRNAs in pathophysiological pathways regulating muscle response to damage and regeneration. However, except for a preliminary study performed on $C X M D J$ dog muscle [26], there is currently no experimental data concerning miRNA status specifically in GRMD dog skeletal muscle. Alternatively, a recent study identified deregulated miRNAs in the serum of GRMD dogs [28]. Although GRMD dogs more closely mimic the human disease than $m d x$ mouse, the lack of data on this large animal model represents a real limitation for the accurate description of the dysregulation of miRNAs in a DMD-like context. Moreover, it is important to fill this gap in our knowledge of the GRMD dog model, in particular with regard to the preclinical evaluation of new therapeutic proposals.

We show that systemic delivery of MuStem cells (which are muscle-resident stem cells isolated from healthy dog based on delayed adhesion properties) represents an attractive avenue for future therapeutic applications in DMD patients. Indeed, allogeneic MuStem cell transplantation in GRMD dogs leads to reduced muscle damage, increased regeneration activity, and a persistent stabilization of clinical status [29]. In a previous study, we revealed the impact of MuStem cell transplantation, with an up-regulation of genes reflecting a sustained enhancement of muscle regeneration [30]. In addition, MuStem cells can act on several other biological pathways implicated in protein degradation mechanisms and energy metabolism, evoking a diffuse impact with a large number of targeted biological processes.

In the present study, we firstly aim at defining the miRNA pattern in the skeletal muscle of 9-month-old GRMD dogs corresponding to an advanced state of the disease. Secondly, we attempt to determine how this pattern could be affected following the intravenous delivery of MuStem cells. We determine, for the first time, that miR-222 displays a differential expression pattern in GRMD dog muscle as shown by its marked upregulation. Using in situ hybridization, we show that miR-206 and miR-486 are mainly expressed in clusters of newly regenerated fibres. In addition, we demonstrate an up-regulation of both miR-133 and miR-222 4 months after MuStem cell transplantation, highlighting their potential use as novel markers for the follow-up of effects 
associated with MuStem cell delivery in a dystrophic context.

\section{Methods}

\section{Ethics statement and animals}

This study was approved by the Ethics Committee on Animal Experimentation of the Pays de la Loire Region (France) in accordance with the guidelines from the French National Research Council for the Care and Use of Laboratory Animals (Permit Number: CEEA.2012.104). All the dogs were obtained from the Centre d'élevage du Domaine des Souches (Mézilles, France), which were kept at the Boisbonne Centre for gene and cell therapy of Oniris (Nantes, France). Fourteen 9-month-old golden retriever dogs were included in the study; five were healthy and nine were GRMD. Affected dogs were identified in the 1st week of life using polymerase chain reaction (PCR)-based genotyping. This identification was corroborated by a dramatic and early rise in levels of serum creatine kinase [31]. GRMD dogs were divided into three subsets: three GRMD dogs received neither an immunosuppressive regimen nor cell transplantation (subset denoted as GRMD), three received only a continuous immunosuppressive regimen (mock GRMD) and the remaining three received MuStem cell transplantation under immunosuppression (GRMD $^{\text {MuStem }}$ ) (Table 1).

\section{Cell delivery procedure}

MuStem cells were isolated from a pool of hindlimb muscles of 2.5-month-old healthy dogs and prepared as previously described [29]. Three GRMD dogs (ranging from 3.5 to 4.5 months of age) were submitted to a continuous cyclosporine-based immunosuppressive regimen as established by Rouger et al. (2011) and received three cell injections (spaced at an interval of 2 weeks): GRMD $^{\text {MuStem }}$. Each of these injections delivered $5.5 \times 10^{7}$ to $8.0 \times 10^{8} \mathrm{MuStem}$ cells $/ \mathrm{kg}$ into the cephalic vein using laminar flow at a rate of $12 \mathrm{~mL} / \mathrm{min}$.

\section{Clinical follow-up}

A clinical score was measured weekly for all GRMD dogs following a previously described method [29, 32]. Dogs were weighted and clinically assessed in a nonblinded manner by a veterinarian on a weekly basis during all the experiment. Briefly, this clinical score is based on 11 locomotion and muscular criteria and 6 items related to general health status. It is expressed as a percentage of the maximum score defined as $100 \%$ for a healthy dog.

\section{Muscle sampling}

Biceps femoris muscle samples $\left(0.5 \mathrm{~cm}^{3}\right.$ fragments) were collected surgically from the middle portion of the muscle in 9-month-old (37 \pm 5 -week-old) healthy, GRMD, mock GRMD and GRMD ${ }^{\text {MuStem }}$ dogs. The Biceps femoris is a large and easily accessible muscle. This time-point corresponds to 4 months after systemic administration to the GRMD ${ }^{\text {MuStem }}$ dogs and is the same as in our previous transcriptomic study [30]. Muscle fragments were divided into two parts for histological and molecular analyses, and subsequently stored at $-80{ }^{\circ} \mathrm{C}$ until processing.

\section{Histological analysis}

Eight $\mu$ m-thick cryosections were incubated with mouse primary antibody directed against the developmental myosin heavy chain (MyHCd, $1 / 20$, Novocastra, Newcastle, United Kingdom) for $1 \mathrm{~h}$ at $37^{\circ} \mathrm{C}$. After successive incubation with a secondary biotinylated antibody and streptavidin horseradish peroxidase conjugate $(1 / 300$, Dako, Glostrup, Denmark), MyHCd protein was visualized by diamidinobenzidine tetrahydrochloride (DAB; Dako). Slides were then dehydrated and mounted in a dry mounting medium. Morphometric analysis was performed using a digital camera (Nikon DXM 1200, Nikon Instruments, Badhoevedorp, The Netherlands) combined with image-analysis software (NIS, Nikon). Microscopic fields were randomly selected on immunolabelled sections using intermediate magnification to observe at least 100 fibres. To determine the percentage of MyHCd ${ }^{+}$fibres, at least 662 fibres $(1030 \pm 125)$ were counted on three randomly selected microscopic fields. For each measurement, reproducibility is better than $92 \%$. For dystrophin labelling, all acquisitions were performed with the same signal amplification resulting from identical detector gain, as previously described [29]. To determine the proportion of dystrophin ${ }^{+}$fibres, $880 \pm 101$ total fibres were counted (laminin red fluorescent immunolabelling) in the Biceps femoris muscle sections of the GRMD $^{\text {MuStem }}$ dogs $(n=3)$ and then the number of fibres

Table 1 Description of the fourteen male dogs included in the miRNA study

\begin{tabular}{|c|c|c|c|c|c|}
\hline Study & Dog group & Number of animals & Phenotype & Immunosuppression & MuStem cell injection \\
\hline \multirow[t]{2}{*}{ Physiopathology } & Healthy & 5 & healthy & None & None \\
\hline & GRMD & 3 & dystrophic & None & None \\
\hline \multirow[t]{2}{*}{ MuStem cell impact } & mock GRMD & 3 & dystrophic & Yes & None \\
\hline & GRMD MuStem & 3 & dystrophic & Yes & Yes \\
\hline
\end{tabular}


expressing dystrophin was determined from DYS2 (Novocastra) green fluorescent immunolabelling.

\section{miRNAs isolation and qPCR}

miRNAs were extracted from muscle samples of right and left Biceps femoris of each animal. The mirVana miRNA isolation kit (Ambion, Austin, TX, USA) was used, according to the manufacturer's instructions, and microRNAs were finally eluted with $100 \mu \mathrm{L}$ of water and quantified using a Nanodrop spectrophotometer (Labtech, Wilmington Delaware, USA). Reverse Transcription reactions were carried out on $5 \mathrm{ng}$ of miRNAs using the TaqMan miRNA Reverse Transcription kit (Applied Biosystems, Foster City, CA, USA) and miRNA-specific stem loop primers for miR-1, miR-133a, miR-206, miR-222, and miR-486 (Applied Biosystems miRNA assays). Real-time PCR reactions were performed at least in duplicate with miRNA-specific primers and Taqman ${ }^{\circledR}$ probes on the CFX96 PCR System (BioRad). Data were normalized using U6 snRNA (RNU6B) as an internal control and differential expression was calculated using the $2^{-\Delta \Delta C t}$ method. For each miRNA, statistical differences between two groups were analysed by a Mann-Whitney test.

\section{In situ hybridization}

In situ hybridization (ISH) was performed on muscles from healthy, GRMD, mock GRMD and GRMD ${ }^{\text {MuStem }}$ dogs, using digoxigenin (DIG)-labelled miRCURY locked nucleic acid (LNA) detection probes (Exiqon, Vedbaek, Denmark), corresponding to hsa-miR-486 (38596-05), hsa-miR-206 (88081-15) and scramble-miR (99004-05 and 99004-15). Ten $\mu \mathrm{m}$-thick frozen muscle sections were air-dried for $1 \mathrm{~h}$ and fixed for $10 \mathrm{~min}$ in $4 \%$ paraformaldehyde. Then, the sections were permeabilized with proteinase $\mathrm{K}(20 \mu \mathrm{g} / \mathrm{mL})$ for $10 \mathrm{~min}$. For prehybridization, the tissue sections were covered for $1 \mathrm{~h}$ with hybridization buffer containing $50 \%$ formamide, 4X SSC, 1X Denhardt's solution, $500 \mu \mathrm{g} / \mathrm{mL}$ salmon sperm DNA (Sigma-Aldrich, Saint Quentin Fallavier, France), $10 \%$ dextran sulfate and 1X Blocking Reagent (Roche, Basel, Switzerland). For hybridization, $50 \mathrm{nM}$ of DIG-labelled probes diluted in hybridization buffer were applied per section and incubated in a sealed humidified chamber for $16 \mathrm{~h}$ at $55{ }^{\circ} \mathrm{C}$. A stringency wash was performed for $30 \mathrm{~min}$ in $50 \%$ formamide/1X SSC, followed by two $0.2 \mathrm{X}$ SSC washes for $15 \mathrm{~min}$ each. Sections were then incubated with alkaline phosphatase-conjugated sheep anti-DIG (1/1000, Roche) antibody for $2 \mathrm{~h}$. Hybridized probes were visualized by color reaction with nitro-blue tetrazolium (NBT) and 5-bromo 5-chloro-3indolyl phosphate (BCIP) overnight at $4{ }^{\circ} \mathrm{C}$. Slides were counterstained with Nuclear Fast Red and mounted in a Vectamount mounting medium (Vector Laboratories,
Burlingame, USA). In situ analysis was carried out by one "blinded" reader and one non-"blinded" reader, yielding comparable results. No signal was detected using scrambled control probes.

\section{Western Blot}

For protein extraction, muscles were homogenized in RIPA lysis buffer containing $150 \mathrm{mM} \mathrm{NaCl}, 50 \mathrm{mM}$ Tris- $\mathrm{HCl} \mathrm{pH}$ 7.4, 1 \% Nonidet-P40, 1 \% glycerol, $1 \mathrm{mM}$ EDTA and protease inhibitors using the Precellys (Ozyme, France) $(2 \times 10 \mathrm{~s}, 6500 \mathrm{rpm})$. Homogenates were centrifuged at $10,000 \mathrm{~g}$ to pellet debris and supernatants were centrifuged at 20,000 g (45 min, $\left.4{ }^{\circ} \mathrm{C}\right)$. Protein concentration was determined using a BCA protein assay (Sigma-Aldrich). Fifty $\mu$ g of proteins of tissue homogenate were resolved by sodium dodecyl sulfate polyacrylamide gel electrophoresis (SDS-PAGE) on 4-12\% polyacrylamide gels (NuPage, Life Technologies, Illkirch, France) and electroblotted onto nitrocellulose membranes (Protran BA 83, GE Healthcare) using a Bio-Rad ${ }^{\circ}$ liquid blotting system at $30 \mathrm{~mA}$ for $2 \mathrm{~h}$. The membranes were blocked using $50 \%$ Blocking Buffer (Odyssey ${ }^{\oplus}$, LiCor Biosciences, Lincoln, NE, USA) in PBS ( $1 \mathrm{~h}$, room temperature) and incubated overnight at $4{ }^{\circ} \mathrm{C}$ with primary antibodies against myosin heavy chain (MHC) MF20 (1/1000, Developmental Studies Hybridoma Bank /DSHB, Iowa City, IA, USA), $\alpha$-actinin (1/1000, SigmaAldrich), MYH7 (1/5000, Abcam, Cambridge, MA, USA). After washing with Tween $0.1 \%$ in PBS, the blots were incubated with horseradish peroxidase-conjugated or fluorophore-conjugated anti-mouse and anti-rabbit secondary antibody. After washing, the samples were coverslipped with Mowiol Mounting Medium (Calbiochem EMD Biosciences, San Diego, CA, USA) and scanned with a blue $488 \mathrm{~nm}$ argon ion laser using the C1 inverted Nikon TE-2000 laser scanning confocal microscope (Nikon, Champigny, France). Equal protein loading was checked through $\alpha$-actinin labelling and Ponceau S staining of the membranes.

\section{Results}

\section{Differential miRNA expression level in GRMD dog muscle}

The expression levels of five miRNAs in healthy and GRMD dog muscles were investigated to determine whether miRNAs (previously shown to be differentially expressed in skeletal muscle of DMD patients and $m d x$ mice) display the same deregulations in the clinically relevant dog model. The generated data are normalized to RNU6B, which is an internal control RNA frequently used in miRNA expression studies, yielding an average Ct of $30.89 \pm 0.93$ (SD) for the whole set of tested samples (Additional file 1: Fig. S1). In the dystrophic context, we observe a markedly increased expression of miR-222 $(p=0.03)$ and a tendency to decreased 
expression of miR-486 ( $p=0.14)$ (Fig. 1a). On the other hand, miR-1, miR-133a, miR-206 expression levels are unchanged in GRMD dogs.

\section{miRNA cellular localization in GRMD dog muscle}

To complete our PCR-based observations, we investigated the subcellular and tissue localization of the

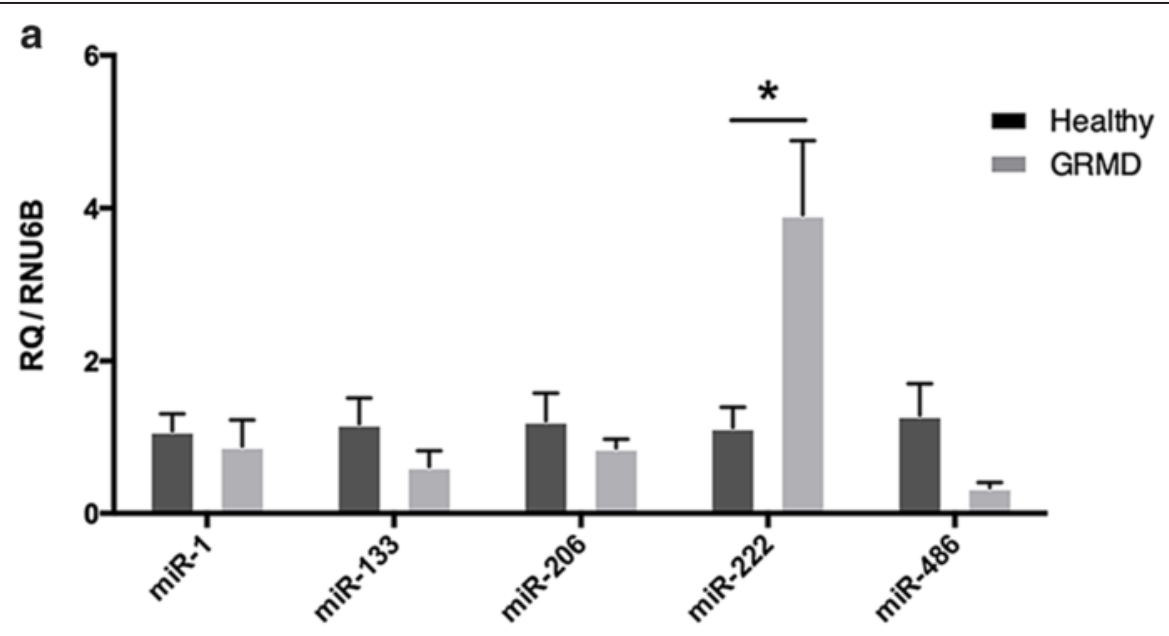

b
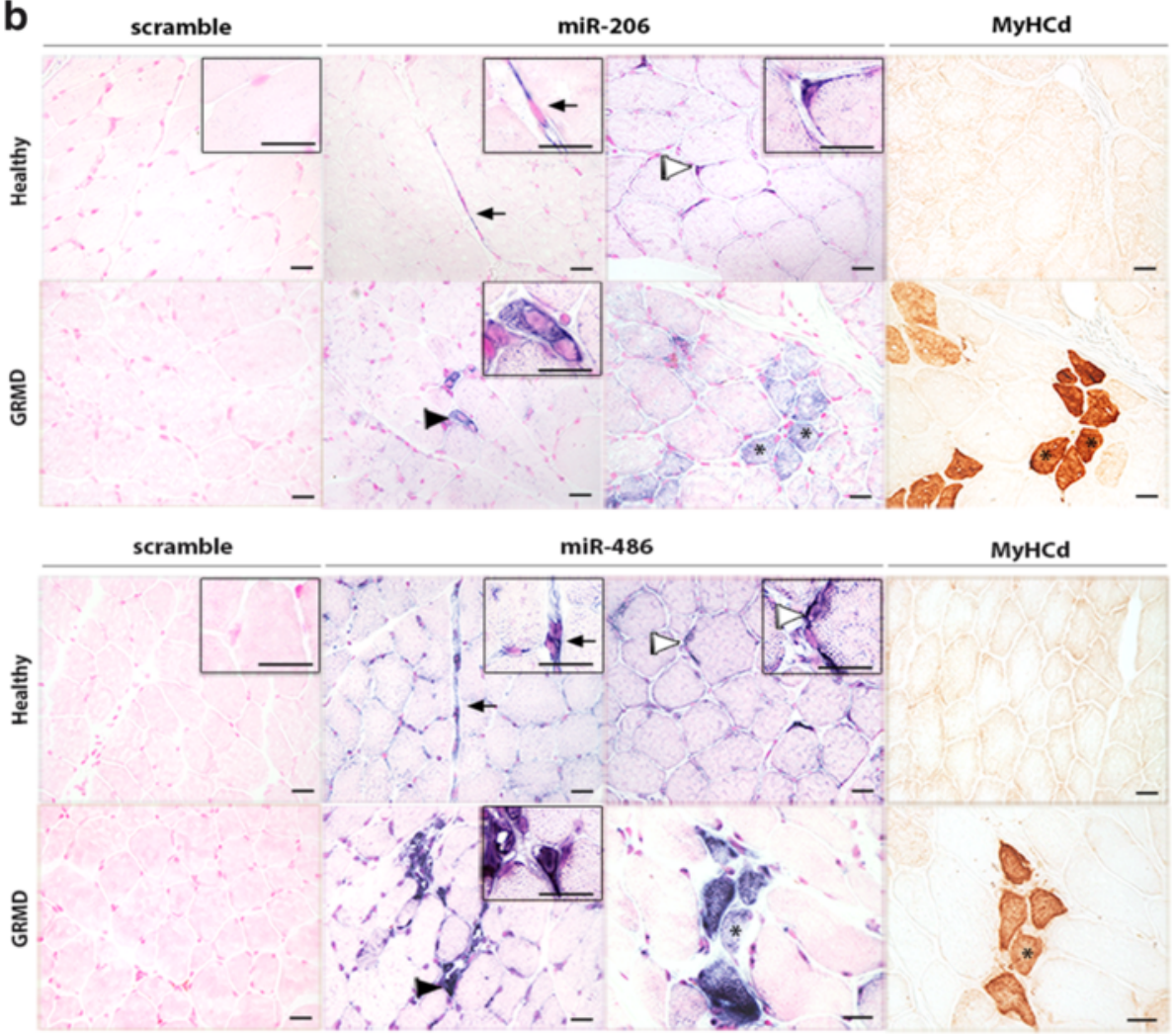

Fig. 1 a Relative expression levels of miRNAs in dog muscle. Expression levels of miR-1, miR-133a, miR-206, miR-222 and miR-486 were determined in 9-month-old healthy $(n=5)$ and GRMD $(n=3)$ dog muscle by real-time PCR and were normalized to RNU6B levels. Results are indicated as relative expression and are presented as mean \pm SEM. ${ }^{*} p<0.05$. $\mathbf{b}$ miR-206 and miR-486 localization in healthy and GRMD dog skeletal muscle. The tissue localization was assayed by in situ hybridization on Biceps femoris muscle cryosections derived from healthy and GRMD dogs, using digoxigenin labelled LNA probes. Representative images are shown. Upper panel: miR-206 expression. In healthy dog muscle, miR-206 expression is detected in cytoplasmic processes of vessel endothelial cells (black arrow) and around some peripheral nuclei (empty arrow head) of muscle fibres. In GRMD dog muscle, a strong signal is detected in myoblasts (black arrow head) and regenerating $\mathrm{MyHCd}^{+}$fibres (asterisk). Lower panel: miR-486 expression. In healthy dog muscle, miR-486 is detected both in endothelial cells (black arrow) and around peripheral nuclei (empty arrow head). In GRMD dog muscle, miR-486 is localized in myoblasts (black arrow head) and regenerating fibres (asterisk). Scale bar $=25 \mu \mathrm{m}$ 
miRNAs in a dystrophic context by using ISH. We successfully performed in situ exploration of two of the five miRNA tested: miR-206, a key modulator of skeletal muscle development and disease, and miR-486, an important factor in the regulation of DMD muscle pathology (Fig. 1b). In healthy dog muscle, miR-206 and miR-486 are expressed around some peripheral nuclei of fibres as well as in endothelial cells. Furthermore, most of the fibres display a perinuclear miR-486 signal. Interestingly, in GRMD dogs, miR-206 and miR-486 are mainly localized in newly formed myoblasts and regenerating fibres, as demonstrated by $\mathrm{MyHCd}^{+}$labelling on serial sections. Unfortunately, we failed to detect significant signal for miR-222 and miR-133a.

\section{Clinical and tissue consequences of the intravenous delivery of MuStem cells}

In the context of the allogeneic transplantation protocol, GRMD $^{\text {MuStem }}$ dogs and mock GRMD dogs were both submitted to immunosuppression. To determine whether cyclosporine treatment on its own can modify the miRNA profile, the expression levels were first determined in GRMD dogs with or without immunosuppression. We observe no significant change except for miR206, which is increased under our immunosuppressive regimen $(p=0.07)$ (Fig. 2). Interestingly, even though miR-206 expression is modified, its subcellular localization remains unchanged (data not shown here).

Mock GRMD dogs display a progressive clinical impairment characterized at 9 months age by a clinical score of $43.5 \% \pm 25.2$. By contrast, all GRMD ${ }^{\text {MuStem }}$

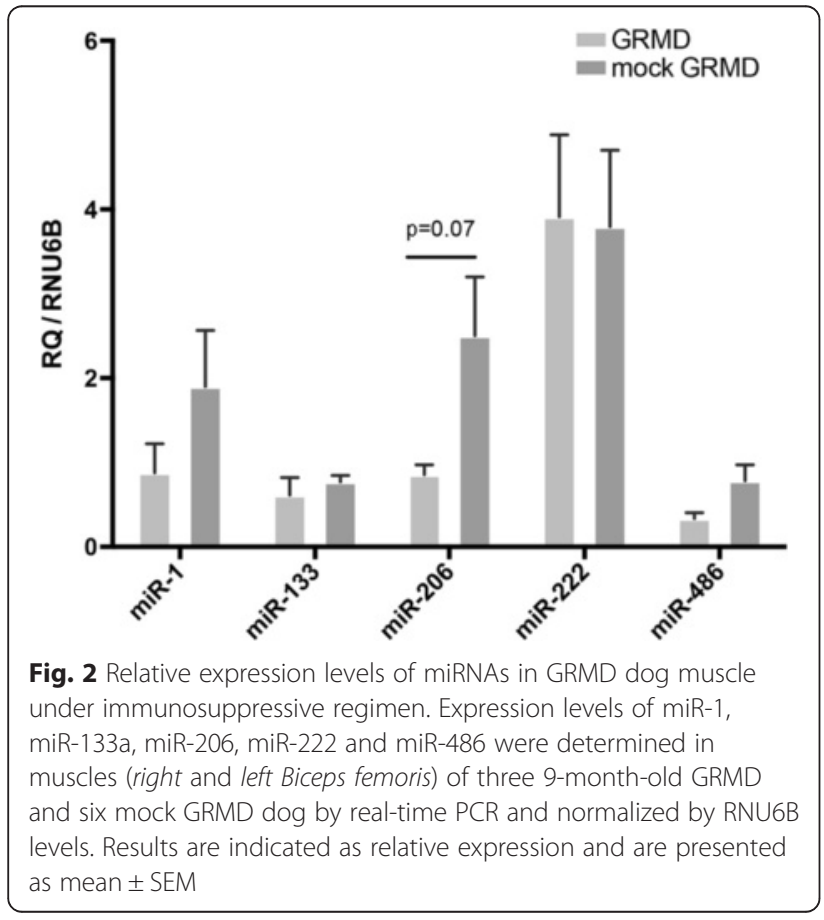

dogs display an early and persistent stabilization of their clinical score that is maintained above $80.9 \% \pm 9.3$ at the same age. Repeated-measures ANOVA carried out from 7.5 to 8.5 months of age indicates a trend towards a positive effect of the MuStem cell delivery $(\mathrm{F}=3926 ; p$ $=0.059$ ) (Additional file 2: Fig. S2). Regenerative activity is also assessed on muscle sections using a specific MyHCd labelling. While $12.9 \% \pm 5.3$ of the fibres express this developmental isoform in mock GRMD dog muscle, the proportion of $\mathrm{MyHCd}^{+}$fibres is $20.5 \% \pm 3.9$ in GRMD ${ }^{\mathrm{MuStem}}$ dogs, thus demonstrating a tendency to an increased muscle regenerative activity following MuStem cell delivery (Fig. 3a).

Immunofluorescent labelling of dystrophin in the $B i$ ceps femoris muscle shows no expression of this protein in mock GRMD dogs, apart from some rare positive fibres corresponding to revertant fibres. Muscle sections of GRMD ${ }^{\mathrm{MuStem}}$ dogs are characterized by a very low expression level of dystrophin compared to that observed in healthy dog muscle (Fig. 3b). Fibres observed in healthy dog are defined by a high fluorescent labelling intensity as well as by continuous labelling all along the fibre membrane. On the contrary, the limited dystrophin expression observed in GRMD ${ }^{\text {MuStem }}$ dog muscle is illustrated by a discontinuous labelling along the membrane and is restricted at the most to $14.4 \%$ of all fibres (Fig. 3b).

\section{Modified muscle miRNA expression following MuStem cell transplantation}

The expression level of five miRNAs was investigated on the GRMD ${ }^{\text {MuStem }}$ dogs to determine whether cell transplantation could have an impact on their expression. We show that miR-133a $(p=0.03)$ and miR-222 $(p=0.03)$ are up-regulated in GRMD $^{\text {MuStem }}$ dogs compared to mock GRMD dogs (Fig. 4a), while miR-1, miR-206 and miR-486 expressions appear unchanged. In terms of tissue distribution, the ISH carried out on the Biceps femoris muscle of GRMD ${ }^{\text {MuStem }}$ dog reveals an expression of miR-486 and miR-206 on clustered $\mathrm{MyHCd}^{+}$regenerative fibres (Fig. 4b), which confirms previous findings [33,34] and reinforces the hypothesis whereby these two miRNAs are unaffected by the infusion of cells. Lastly, while miR-222 is up-regulated in GRMD versus healthy dog muscle, it is found to be even more up-regulated in GRMD $^{\mathrm{MuStem}}$ versus mock GRMD dog muscle $(p=0.03)$ (Fig. 4a). To corroborate this result, we investigated the protein abundance of miR-222 targets (sarcomeric proteins: myosin heavy chain (MHC) and MYH7). We observe a decrease in sarcomeric myosin heavy chain proteins expression in GRMD ${ }^{\mathrm{MuStem}} \mathrm{dog}$, reflecting the inhibition of sarcomeric protein accumulation concordant with miR-222 overexpression [20] (Fig. 5a and b). 


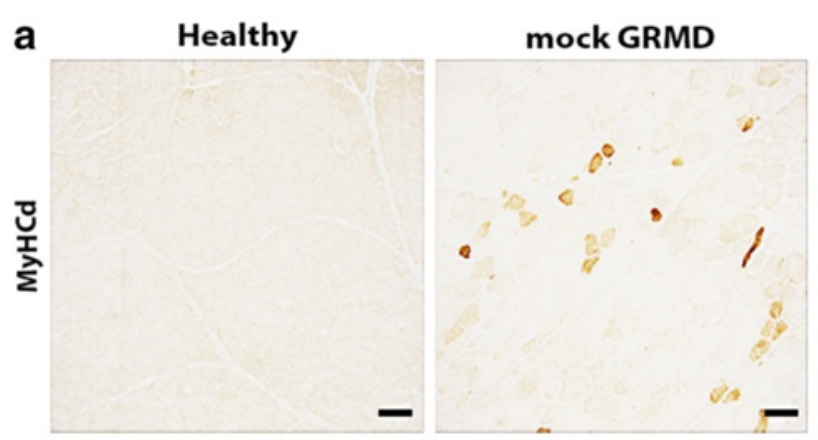

b
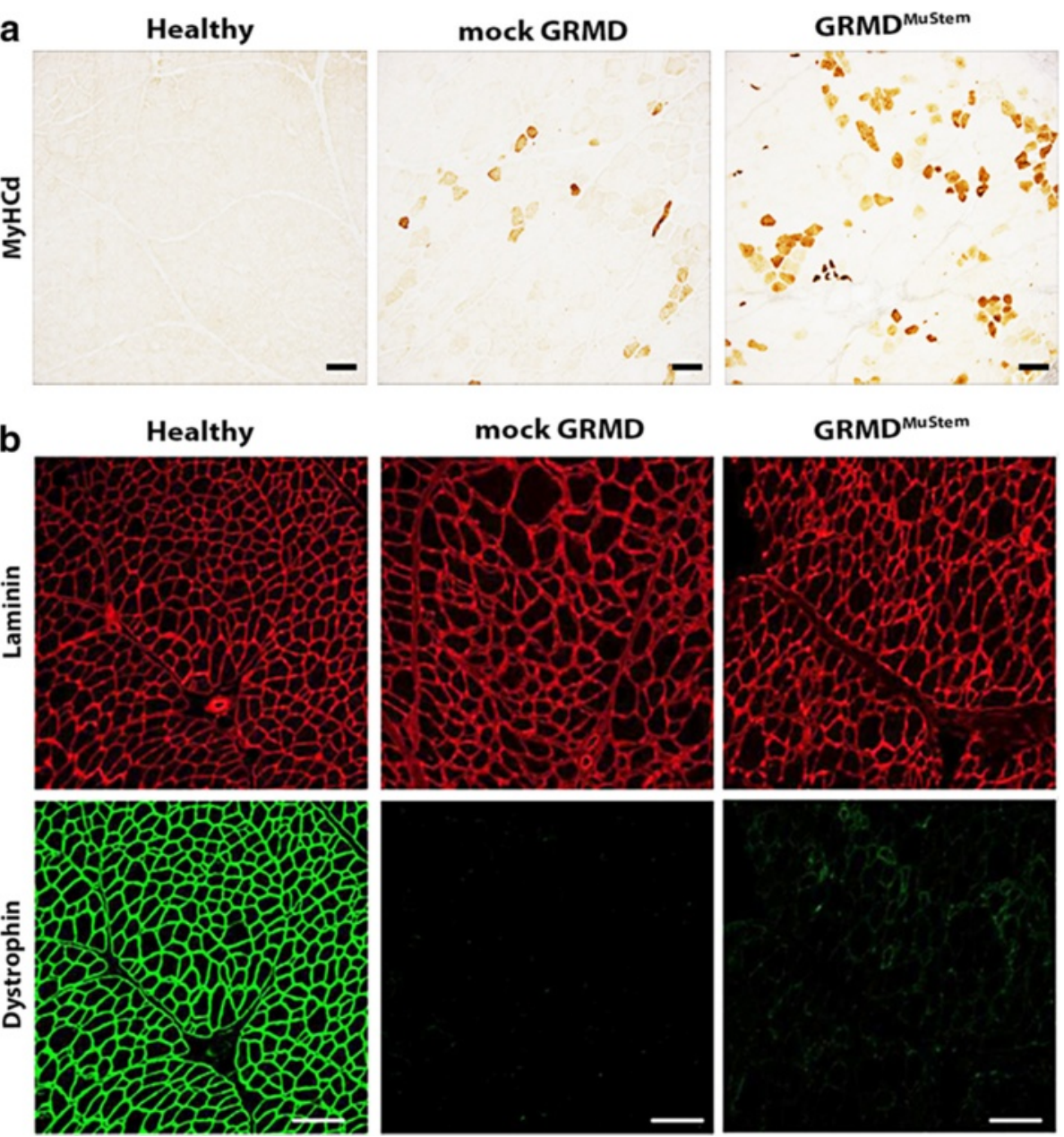

GRMD ${ }^{\text {Mustem }}$
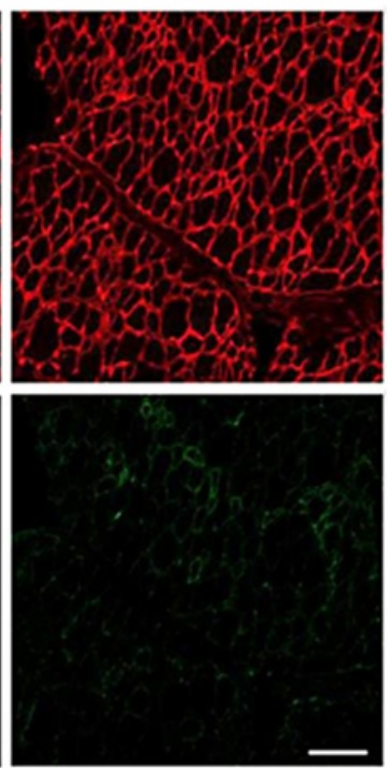

Fig. 3 a MyHCd immunolabelling in healthy, mock GRMD and GRMD ${ }^{\text {Mustem }}$ dogs. Transverse cryosections of the Biceps femoris muscle of 9-month-old healthy, mock GRMD and GRMD ${ }^{\text {MuStem }}$ dogs. Scale bar $=50 \mu \mathrm{m}$. b Dystrophin immunolabelling in healthy, GRMD and GRMD ${ }^{\text {MuStem }}$ dogs. Transverse cryosections of the Biceps femoris muscle of 9-month-old healthy, mock GRMD and GRMDMustem dogs. The laminin (red) and dystrophin (green) fluorescent immunolabellings are presented. Scale bar $=200 \mu \mathrm{m}$

\section{Discussion}

miRNAs are considered as integral components of the regulatory circuitry for myogenesis, even if their full role in muscle growth and development remains to be elucidated $[13-15,18]$. Numerous studies provide increasing evidence for the involvement of miRNAs in myopathies, and particularly in muscular dystrophies [22, 23, 35]. It has been recently reported that miRNAs are promising biomarkers for monitoring disease progression [28, 36, 37]. In this regard, several serum miRNAs have been identified as dysregulated in GRMD dogs, using a high-throughput miRNA sequencing screening [28]. Based on the clinical relevance of the animal model used, these results have allowed authors to select these miRNAs as candidate biomarkers for DMD patients. In addition, it is increasingly acknowledged that miRNAs could represent useful tools in the assessment of experimental therapies to cure muscle diseases $[25,28,38]$. Nevertheless, further investigations need to be conducted to identify the role of these dysregulated miRNAs in muscle pathophysiology.

Up to now, most of the results presented on muscle miRNAs have been obtained from the $m d x$ mouse model, which is known to show limitations for the study of pathogenetic mechanisms and therapeutic trials specific to DMD. For this reason, we aim at establishing, for the first time, a description of miRNA dysregulations in GRMD dog skeletal muscle based on a dedicated set: miR-1, miR-133a, miR-206, miR-222 and miR-486. In accordance with previous observations made in the $m d x$ mouse model and DMD patients [10, 22, 25, 26], we find that miR-222 and miR-486 exhibit a marked upregulation and a down-regulation in 9-month-old GRMD dog muscle, respectively. On the contrary, RTqPCR performed on Biceps femoris muscle extract fails to reveal any dysregulation of miR-206, in contrast with the previously described up-regulation in both the $m d x$ 

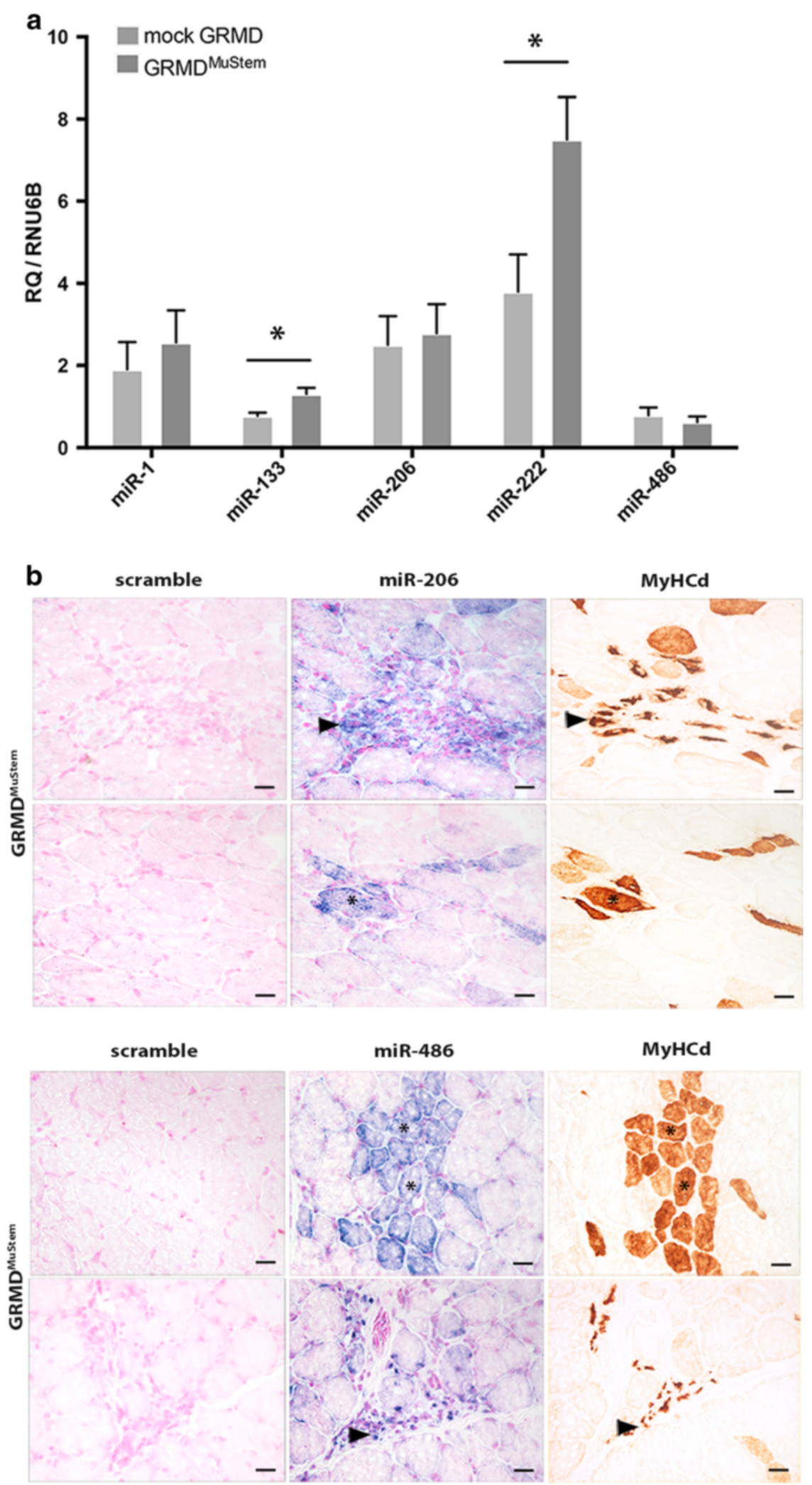

MyHCd

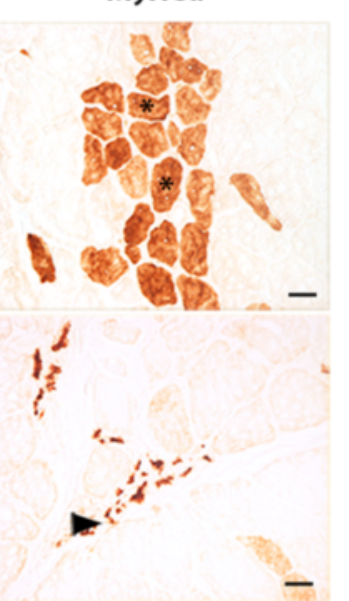

Fig. 4 (See legend on next page.) 
(See figure on previous page.)

Fig. 4 a Modulation of miRNA levels in GRMD dog muscles after systemic administration of MuStem cells. Expression levels of miR-1, miR-133a, miR-206, miR-222, and miR-486 were determined in muscles (right and left Biceps femoris) of six 9-month-old GRMDMustem dogs compared to six mock GRMD dogs. RNU6B was used as internal control and relative expressions are presented as mean $\pm S E M .{ }^{*} p<0.05$. b miRNA localization in GRMD dog muscle after MuStem cell transplantation. Upper panel: miR-206 expression. Lower panel: miR-486 expression. miR-206 and miR-486 are detected in myoblasts (black arrow head) and in the cytoplasm of small and intermediate regenerating MyHCd fibres (asterisk). Scale bar $=25 \mu \mathrm{m}$

mouse model and DMD patients [10, 22, 25, 26]. Nevertheless, up-regulation of miR-206 is not observed in all dystrophic muscles. Indeed, McCarthy et al. demonstrated that miR-206 is overexpressed in the most severely affected $m d x$ muscles, i.e. the diaphragm, but not in the hindlimb [27]. In addition, Yuasa et al. showed a decreased expression of this miRNA in the CXMDJ tibialis anterior muscle compared to the control [26]. Our results support Yuasa's hypothesis that increased expression of miR-206 in $m d x$ muscle may reflect active and efficient regeneration, whereas its decreased expression in $C X M D J$ muscle may illustrate relatively exhausted regeneration potential [26]. In the present study, we use in situ detection to obtain original information concerning the muscle tissue distribution of the miRNAs, thus improving the characterization of their tissue expression. Combined in situ hybridization and MyHCd labelling demonstrate that miR-206 is histologically related to muscle fibre regenerative processes in GRMD dog, being mainly expressed in newly formed fibres. Interestingly, this distribution has been previously reported in $m d x$ muscles $[10,26]$ that have considerable regenerative capacity $[39,40]$.

While we describe here the expression patterns of a miRNA subset, further studies are required to understand the implication of miRNAs in the pathophysiology of GRMD dog. In this paper, we show that a continuous cyclosporine-based immunosuppressive regimen maintained over a period of 5 months does not lead to a major modification of the investigated miRNAs levels, except for miR-206 that tends to increase. This result highlights a selective impact of immunosuppression treatment on the expression levels of miRNAs, thus strongly suggesting that the immunosuppressive component must be considered in the assessment of allogeneic cell-based preclinical studies requiring the use of immunosuppression [41].

In the present study, we attempt to determine whether the systemic delivery of MuStem cells, which increases muscle regenerative activity and stabilizes the clinical status of GRMD dogs [29], can concomitantly affect the expression levels of miRNAs that are able to modulate key cellular processes at a post-transcriptional level. This hypothesis seems particularly interesting because the observed clinical and tissue benefits following MuStem cell infusion are linked to a low dystrophin protein level as well as a limited percentage of dystrophin-positive fibres, clearly evoking the implication of other molecular pathways $[29,30]$. Firstly, it is surprising that the expression levels of miR-206 and miR-486 (two miRNAs known to be implicated in the regenerative process) are not upregulated in transplanted GRMD dogs. It could be hypothesized that the increased regenerative potential revealed in GRMD ${ }^{\mathrm{MuStem}} \operatorname{dogs} 4$ to 5 months after

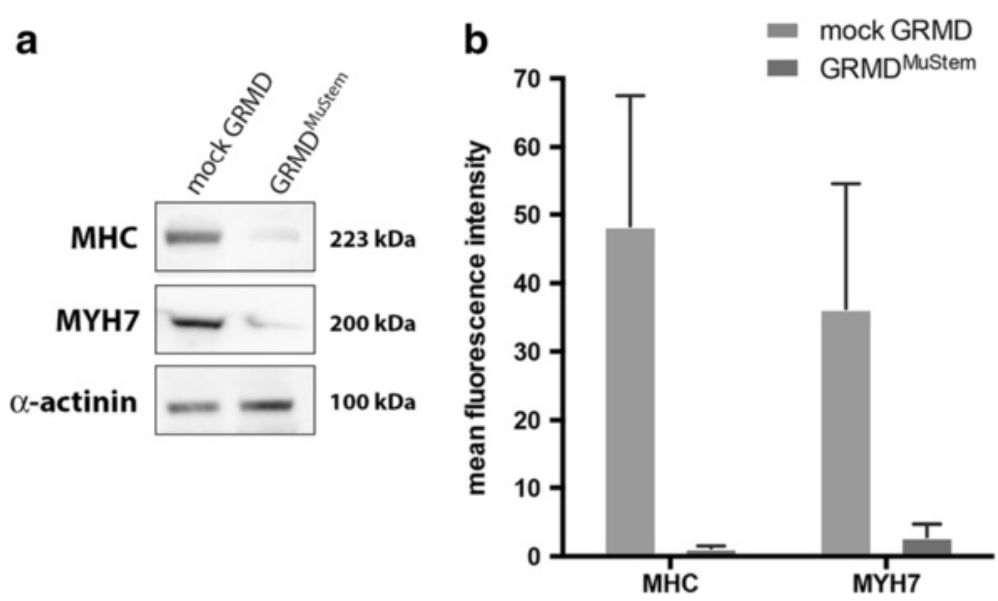

Fig. 5 a MHC and MYH7 protein expressions in mock GRMD compared to GRMD dog after MuStem cell transplantation. a Representative western blot analyses. a-actinin is used as control. b Graphical representation of immunoblot analyses in three mock GRMD and GRMDMustem samples. The mean fluorescence intensity is represented along with the SEM (standard error of the mean) for the different samples 
transplantation is not sufficient to be associated with a differential expression of miR-206. Secondly, we demonstrate an up-regulation of miR-133a and miR-222 expression after systemic delivery of MuStem cells. Interestingly, changes of these miRNAs are reported to be implicated in the disruption of sarcomere organization [20, 42]. Expression of miRNA-222 in myoblasts induces myogenin expression followed by inhibition of sarcomeric protein accumulation. Our finding on the down-expression of two sarcomeric proteins MYH7 and MHC in GRMD ${ }^{\text {Mustem }}$ muscle suggests that miR-133a and miR-222 could be involved in the remodelling of the sarcomeric assembly, thus preventing the accumulation of sarcomeric component aggregates observed in dystrophic muscle. Moreover, the pathway analysis performed to provide functional annotation based on KEGG terms (DIANA-miRPath) shows an enrichment of miR-133 in many pathways linked to ubiquitin mediated proteolysis as well as regulation of the actin cytoskeleton. Also, this indicates that miR-222 is involved in the molecular pathways linked to the cell cycle, the insulin signalling pathway and ubiquitin mediated proteolysis.

These observations corroborate our previous study [30] in which we demonstrated that systemic administration of MuStem cells greatly enhances ubiquitinmediated protein degradation and induces insulin resistance in skeletal muscle.

\section{Conclusion}

In the present study, we characterize the muscle expression pattern of five relevant miRNAs in the GRMD dog model. Interestingly, we define a specific global miRNA signature distinct from those found in the $m d x$ mouse model but also in DMD patients. In addition, we establish that MuStem cell infusion is characterized by an upregulation of both miR-133a and miR-222, positioning them as potential useful markers to assess the efficacy of a cell-based strategy. Further functional studies and target exploration should be carried out to improve our understanding of the links with MuStem cell-associated effects.

\section{Ethics approval}

This study was approved by the Ethics Committee on Animal Experimentation of the Pays de la Loire Region, France, in accordance with the guidelines from the French National Research Council for the Care and Use of Laboratory Animals (Permit Number: CEEA.2012.104).

\section{Consent for publication}

Not applicable.

\section{Availability of data and materials}

All the data supporting the findings are contained within the manuscript.

\section{Additional files}

Additional file 1: Figure S1. Validation of RNU6B as an internal control for study of miRNAs expression in dog muscles. The threshold cycle (CT) of RNU6B was determined in each sample and shows similar levels in healthy, GRMD, mock GRMD and GRMD ${ }^{\text {MuStem }}$ dogs. (TIF 239 kb)

Additional file 2: Figure S2. Clinical follow-up. Clinical scores of mock GRMD dogs (-) and MuStem cell-injected dogs (-) are represented as mean \pm SD. The clinical score of each GRMD dog was assessed weekly and expressed as a percentage of a theoretical healthy dog score. Limits of the MuStem cell delivery window are indicated (dashed lines). (PDF $32 \mathrm{~kb}$ )

\section{Abbreviations}

DMD: Duchenne muscular dystrophy; GRMD: Golden Retriever muscular dystrophy; GRMD MuStem: GRMD dogs transplanted with MuStem cells; ISH: in situ hybridization; MHC: Myosin Heavy Chain; miRNAs: microRNAs;

MyHCd: developmental Myosin Heavy Chain.

\section{Competing interests}

The authors declare that they have no competing interests.

\section{Authors' contributions}

FR designed and performed the miRNA quantification experiments, drafted the manuscript and prepared the figures. CB designed and carried out the in situ hybridization, performed the miRNA quantification experiments, drafted the manuscript and prepared the figures. TL performed clinical follow-up of the dogs, tissue sampling and described the in situ hybridization experiments. LD directed and interpreted the immunofluorescence and laser scanning confocal analysis experiments. ML and HG performed the tissue sampling, imunohistochemistry experiments and morphometric analyses. KR and LG designed the set of experiments, analysed the data and critically revised the manuscript. All authors contributed to the final draft of the manuscript and approved the final version.

\section{Acknowledgements}

The authors would like to thank the staff of the Boisbonne Centre (Oniris, Nantes, France) for the handling and care of the GRMD dog colony and Prof. J-Y. Deschamps and Dr. S. Moullec for the clinical surgery; I. Leroux and C. Schleder for MuStem cell isolation; A. Lardenois for helpful discussions; C. Le Guiner for providing some GRMD muscle samples. Dr M.S.N. Carpenter for post-editing the English style.

\section{Funding}

This research was carried out in the context of the $\mathrm{HU}$-Cesti project that receives French government financial support managed by the National Research Agency via the investment in the future programme ANR-10-IBHU005. The IHU-Cesti project is also supported by Nantes Metropole and the Pays de la Loire Region. This work was also supported by grants from the FEDER (Fonds Européen de Développement Régional N³7,085) and the Association Française contre les Myopathies (AFM N ${ }^{\circ} 14,379$ ).

\section{Author details}

${ }^{1}$ INRA UMR 703 PAnTher "Physiopathologie Animale et bioThérapie du muscle et du système nerveux", Oniris, Atlanpôle - La Chantrerie, Route du Gachet C.S. 40706, F-44307 Nantes Cedex 03, France. ' LUNAM Université, Oniris, École nationale vétérinaire, agro-alimentaire et de l'alimentation Nantes-Atlantique, F-44307 Nantes, France. ${ }^{3}$ Université de Nantes, F-44322 Nantes, France.

Received: 19 January 2016 Accepted: 3 May 2016

Published online: 11 May 2016

\section{References}

1. Emery AE. Population frequencies of inherited neuromuscular diseases-a world survey. Neuromuscul Disord. 1991;1:19-29.

2. Bonilla E, Samitt CE, Miranda AF, Hays AP, Salviati G, DiMauro S, Kunkel LM, Hoffman EP, Rowland LP. Duchenne muscular dystrophy: deficiency of dystrophin at the muscle cell surface. Cell. 1988;54:447-52. 
3. Cooper BJ, Winand NJ, Stedman H, Valentine BA, Hoffman EP, Kunkel LM, Scott $\mathrm{MO}$, Fischbeck KH, Kornegay JN, Avery RJ. The homologue of the duchenne locus is defective in X-linked muscular dystrophy of dogs. Nature. 1988;334:154-6.

4. Dubowitz V. Neuromuscular disorders in childhood. Old dogmas, new concepts. Arch Dis Child. 1975;50:335-46.

5. Pichavant C, Aartsma-Rus A, Clemens PR, Davies KE, Dickson G, Takeda S, Wilton SD, Wolff J a, Wooddell Cl, Xiao X, Tremblay JP. Current status of pharmaceutical and genetic therapeutic approaches to treat DMD. Mol Ther. 2011;19:830-40.

6. Kornegay JN, Bogan JR, Bogan DJ, Childers MK, Li J, Nghiem P, Detwiler DA, Larsen CA, Grange RW, Bhavaraju-Sanka RK, Tou S, Keene BP, Howard JF, Wang J, Fan Z, Schatzberg SJ, Styner MA, Flanigan KM, Xiao X, Hoffman EP. Canine models of duchenne muscular dystrophy and their use in therapeutic strategies. Mamm Genome. 2012;23:85-108.

7. Guevel L, Lavoie JR, Perez-Iratxeta C, Rouger K, Dubreil L, Feron M, Talon S, Brand $M$, Megeney $L$ a. Megeney $L$ a: Quantitative proteomic analysis of dystrophic dog muscle. J Proteome Res. 2011;10:2465-78.

8. Nghiem PP, Hoffman EP, Mittal P, Brown KJ, Schatzberg SJ, Ghimbovschi S, Wang Z, Kornegay JN. Sparing of the dystrophin-deficient cranial sartorius muscle is associated with classical and novel hypertrophy pathways in GRMD dogs. Am J Pathol. 2013;183:1411-24

9. Doran P, Wilton SD, Fletcher S, Ohlendieck K. Proteomic profiling of antisense-induced exon skipping reveals reversal of pathobiochemical abnormalities in dystrophic mdx diaphragm. Proteomics. 2009;9:671-85.

10. Cacchiarelli D, Martone J, Girardi E, Cesana M, Incitti T, Morlando M, Nicoletti C, Santini T, Sthandier O, Barberi L, Auricchio A, Musarò A, Bozzoni I. MicroRNAs involved in molecular circuitries relevant for the duchenne muscular dystrophy pathogenesis are controlled by the dystrophin/nNOS pathway. Cell Metab. 2010;12:341-51.

11. Reinhart BJ, Slack FJ, Basson M, Pasquinelli AE, Bettinger JC, Rougvie AE, Horvitz HR, Ruvkun G. The 21-nucleotide let-7 RNA regulates developmental timing in caenorhabditis elegans. Nature. 2000;403:901-6.

12. Chen K, Rajewsky N. The evolution of gene regulation by transcription factors and microRNAs. Nat Rev Genet. 2007;8:93-103.

13. Goljanek-Whysall K, Pais H, Rathjen T, Sweetman D, Dalmay T, Münsterberg A. Regulation of multiple target genes by miR-1 and miR-206 is pivotal for C2C12 myoblast differentiation. J Cell Sci. 2012;125(Pt 15):3590-600.

14. Kim HK, Lee YS, Sivaprasad U, Malhotra A, Dutta A. Muscle-specific microRNA miR-206 promotes muscle differentiation. J Cell Biol. 2006;174:677-87.

15. Chen J-F, Mandel EM, Thomson JM, Wu Q, Callis TE, Hammond SM, Conlon FL, Wang D-Z. The role of microRNA-1 and microRNA-133 in skeletal muscle proliferation and differentiation. Nat Genet. 2006;38:228-33.

16. Chen J-F, Tao Y, Li J, Deng Z, Yan Z, Xiao X, Wang D-Z. microRNA-1 and microRNA-206 regulate skeletal muscle satellite cell proliferation and differentiation by repressing Pax7. J Cell Biol. 2010;190:867-79.

17. Rao PK, Kumar RM, Farkhondeh M, Baskerville S, Lodish HF. Myogenic factors that regulate expression of muscle-specific microRNAs. Proc Natl Acad Sci U S A. 2006:103:8721-6.

18. Liu N, Williams AH, Kim Y, McAnally J, Bezprozvannaya S, Sutherland LB, Richardson J a, Bassel-Duby R, Olson EN. An intragenic MEF2-dependent enhancer directs muscle-specific expression of microRNAs 1 and 133. Proc Natl Acad Sci U S A. 2007;104:20844-9.

19. Wei W, He H-B, Zhang W-Y, Zhang H-X, Bai J-B, Liu H-Z, Cao J-H, Chang K-C, Li X-Y, Zhao S-H. miR-29 targets Akt3 to reduce proliferation and facilitate differentiation of myoblasts in skeletal muscle development. Cell Death Dis. 2013;4, e668.

20. Cardinali B, Castellani L, Fasanaro P, Basso A, Alemà S, Martelli F, Falcone G. Microrna-221 and microrna-222 modulate differentiation and maturation of skeletal muscle cells. PLoS One. 2009;4, e7607.

21. Dey BK, Gagan J, Dutta A. miR-206 and -486 induce myoblast differentiation by downregulating Pax7. Mol Cell Biol. 2011;31:203-14.

22. Greco S, De Simone M, Colussi C, Zaccagnini G, Fasanaro P, Pescatori M, Cardani R, Perbellini R, Isaia E, Sale P, Meola G, Capogrossi MC, Gaetano C, Martelli F. Common micro-RNA signature in skeletal muscle damage and regeneration induced by duchenne muscular dystrophy and acute ischemia. FASEB J. 2009;23:3335-46.

23. Eisenberg I, Alexander MS, Kunkel LM. MiRNAS in normal and diseased skeletal muscle. J Cell Mol Med. 2009;13:2-11.

24. Erriquez D, Perini G, Ferlini A. Non-coding RNAs in muscle dystrophies. Int J Mol Sci. 2013;14:19681-704.

25. Roberts TC, Blomberg KEM, McClorey G, El Andaloussi S, Godfrey C, Betts C, Coursindel T, Gait MJ, Smith CIE, Wood MJ a. Expression analysis in multiple muscle groups and serum reveals complexity in the microRNA transcriptome of the mdx mouse with implications for therapy. Mol Ther Nucleic Acids. 2012;1(May), e39.

26. Yuasa K, Hagiwara Y, Ando M, Nakamura A, Takeda S, Hijikata T. MicroRNA206 is highly expressed in newly formed muscle fibers: implications regarding potential for muscle regeneration and maturation in muscular dystrophy. Cell Struct Funct. 2008;33:163-9.

27. McCarthy JJ, Esser K a, Andrade FH. MicroRNA-206 is overexpressed in the diaphragm but not the hindlimb muscle of mdx mouse. Am J Physiol Cell Physiol. 2007;293:C451-7.

28. Jeanson-Leh L, Lameth J, Krimi S, Buisset J, Amor F, Le Guiner C, Barthélémy I, Servais L, Blot S, Voit T, Israeli D: Serum Profiling Identifies Novel Muscle miRNA and Cardiomyopathy-Related miRNA Biomarkers in Golden Retriever Muscular Dystrophy Dogs and Duchenne Muscular Dystrophy Patients. Am J Pathol. 2014;184(11):2885-98.

29. Rouger K, Larcher T, Dubreil L, Deschamps J-Y, Le Guiner C, Jouvion G, Delorme B, Lieubeau B, Carlus M, Fornasari B, Theret M, Orlando P, Ledevin M, Zuber C, Leroux I, Deleau S, Guigand L, Testault I, Le Rumeur E, Fiszman M, Chérel Y. Systemic delivery of allogenic muscle stem cells induces longterm muscle repair and clinical efficacy in duchenne muscular dystrophy dogs. Am J Pathol. 2011;179:2501-18.

30. Robriquet F, Lardenois A, Babarit C, Larcher T, Dubreil L, Leroux I, Zuber C, Ledevin M, Deschamps J-Y, Fromes Y, Cherel Y, Guevel L, Rouger K. Differential gene expression profiling of dystrophic Dog muscle after MuStem cell transplantation. PLoS One. 2015;10, e0123336.

31. Honeyman K, Carville KS, Howell JM, Fletcher S, Wilton SD. Development of a snapback method of single-strand conformation polymorphism analysis for genotyping golden retrievers for the X-linked muscular dystrophy allele. Am J Vet Res. 1999;60:734-7.

32. Thibaud J-L, Monnet A, Bertoldi D, Barthélémy I, Blot S, Carlier PG. Characterization of dystrophic muscle in golden retriever muscular dystrophy dogs by nuclear magnetic resonance imaging. Neuromuscul Disord. 2007;17:575-84.

33. Liu N, Williams AH, Maxeiner JM, Bezprozvannaya S, Shelton JM, Richardson JA, Bassel-Duby R, Olson EN. microRNA-206 promotes skeletal muscle regeneration and delays progression of Duchenne muscular dystrophy in mice. J Clin Invest. 2012:122:2054-65.

34. Alexander MS, Casar JC, Motohashi N, Myers J a, Eisenberg I, Gonzalez RT, Estrella E a, Kang PB, Kawahara G, Kunkel LM. Regulation of DMD pathology by an ankyrin-encoded miRNA. Skelet Muscle. 2011;1:27.

35. Eisenberg I, Eran A, Nishino I, Moggio M, Lamperti C, Amato AA, Lidov HG, Kang PB, North KN, Mitrani-Rosenbaum S, Flanigan KM, Neely LA, Whitney $D$, Beggs AH, Kohane IS, Kunkel LM. Distinctive patterns of microRNA expression in primary muscular disorders. Proc Natl Acad Sci U S A. 2007; 104:17016-21

36. Cacchiarelli D, Legnini I, Martone J, Cazzella V, D'Amico A, Bertini E, Bozzoni I. MiRNAs as serum biomarkers for duchenne muscular dystrophy. EMBO Mol Med. 2011:3:258-65.

37. Zaharieva IT, Calissano M, Scoto M, Preston M, Cirak S, Feng L, Collins J, Kole R, Guglieri M, Straub V, Bushby K, Ferlini A, Morgan JE, Muntoni F. Dystromirs as serum biomarkers for monitoring the disease severity in duchenne muscular dystrophy. PLoS One. 2013;8, e80263.

38. Koutsoulidou A, Kyriakides TC, Papadimas GK, Christou Y, Kararizou E, Papanicolaou EZ, Phylactou $L$ a. Elevated muscle-specific miRNAs in serum of myotonic dystrophy patients relate to muscle disease progress. PLoS One. 2015;10:e0125341.

39. Coulton GR, Curtin NA, Morgan JE, Partridge TA. The mdx mouse skeletal muscle myopathy: II. Contractile properties. Neuropathol Appl Neurobiol. 1988;14:299-314.

40. Tanabe $Y$, Esaki K, Nomura T. Skeletal muscle pathology in $X$ chromosome-linked muscular dystrophy (mdx) mouse. Acta Neuropathol. 1986;69:91-5.

41. Barthélémy I, Uriarte A, Drougard C, Unterfinger Y, Thibaud J-L, Blot S. Effects of an immunosuppressive treatment in the GRMD dog model of duchenne muscular dystrophy. PLoS One. 2012;7, e48478.

42. Mishima Y, Abreu-Goodger C, Staton A a, Stahlhut C, Shou C, Cheng C, Gerstein M, Enright AJ, Giraldez AJ. Zebrafish miR-1 and miR-133 shape muscle gene expression and regulate sarcomeric actin organization. Genes Dev. 2009;23:619-32. 